\title{
Neonatal acute liver failure: a diagnosis challenge
}

\author{
Mirta Ciocca, M.D. ${ }^{a}$ and Fernando Álvarez, M.D. ${ }^{b}$
}

\begin{abstract}
Neonatal acute liver failure is a rare, very severe disease with a high rate of mortality. It is clinically and etiologically different from acute liver failure seen in older children and adults. Coagulopathy with an international normalized ratio $\geq 3$ is the critical parameter that defines it. The most common causes are fetal alloimmune hepatitis, previously called neonatal hemochromatosis, viral infections, metabolic disorders, and hemophagocytic lymphohistiocytosis. There is a group of treatable diseases that require a very early diagnosis for the prescription of an adequate treatment. Patients should be immediately referred to a specialized facility where pediatric liver transplantation is available to implement such therapeutic alternative, if indicated.

Key words: neonatal liver failure, congenital alloimmune hepatitis, metabolic disorders, liver transplantation.
\end{abstract}

http:/ /dx.doi.org/10.5546/aap.2017.eng.175

To cite: Ciocca M, Álvarez F. Neonatal acute liver failure: a diagnosis challenge. Arch Argent Pediatr 2017; 115(2):175-180.

\section{INTRODUCTION}

Neonatal acute liver failure (NALF) in the first 30 days of life is a rare, very severe disease with a high mortality rate, which is clinically and etiologically different from acute liver

a. Division of Hepatology and Pediatric Liver Transplant Hospital Alemán.

Buenos Aires.

b. Department of Pediatrics. Centre hospitalier universitaire SainteJustine. University of Montreal. Canada.

E-mail address:

Mirta Ciocca, M.D. mciocca@intramed.net

Funding: None.

Conflict of interest: None.

Received: 7-30-2016. Accepted: 9-10-2016 failure (ALF) seen in older children and adults.

The most common causes of NALF are fetal alloimmune hepatitis $(\mathrm{FAH})$, previously referred to as neonatal hemochromatosis $(\mathrm{NH})$, viral infections, metabolic disorders, and hemophagocytic lymphohistiocytosis (HLH).

The clinical presentation of NALF varies and includes food rejection, growth failure, hypoglycemia, coagulopathy unresponsive to vitamin $\mathrm{K}$, and cholestatic jaundice. It is critical to quickly identify the treatable causes of these conditions, such as galactosemia, fatty acid oxidation disorders, hereditary fructose intolerance, herpes virus infection, etc. ${ }^{1-3}$

\section{Definition}

In adult patients, ALF is characterized by the presence of encephalopathy in the 8 weeks following the onset of clinical jaundice and in the absence of a previously diagnosed liver disease. Detecting hepatic encephalopathy in young children is hard, and it is even practically impossible in newborn infants. For this reason, the Pediatric Acute Liver Failure Study Group included coagulopathy (prothrombin time $\geq 20$ seconds or an international normalized ratio [INR] $\geq 2$ following the administration of parenteral vitamin $\mathrm{K}$ ) as the fundamental finding to define ALF in young children. It should be considered that, in relation to NALF, an INR of up to 2 may be observed in a normal newborn infant, and an INR $\geq 2$ in preterm infants. For this reason, an INR $\geq 3$ should be considered for the definition of NALF. ${ }^{2,3}$

\section{ETIOLOGIES}

\section{Fetal alloimmune hepatitis}

FAH is the most common cause of NALF. It is a liver condition associated with extrahepatic siderosis in different tissues, sparing the reticuloendothelial system. FAH is not considered a disease in itself but the phenotype of a severe fetal liver condition of multiple causes. Liver damage may be the consequence of transplacental passage of maternal antibodies against fetal hepatocyte antigens. In FAH, iron transplacental passage is altered due to a reduction in hepcidin produced by the fetal liver. This hormone controls ferroportin function at the syncytiotrophoblast level and reduces ferrous iron passage to the fetus. The tissues most commonly affected by siderosis are the pancreatic acinar cells, the myocardium, the thyroid follicular epithelium, the adrenal 
cortex, and oropharyngeal and upper respiratory tract mucous glands. ${ }^{1,2}$

FAH may include a significant obstetric history, e.g., oligohydramnios, reduced fetal movement, placental edema, intrauterine growth restriction, and a history of miscarriage or stillbirth. FAH is the prototype of fetal liver disease, which takes place in the neonatal period in the form of NALF. Patients develop severe necroinflammatory activity of the hepatic parenchyma in the first days of life with liver failure. A high rate of recurrence, near $80 \%$, has been reported in subsequent pregnancies after the index case. The disease has been observed in mothers with different partners, but not otherwise. The fact that no cases have been detected among descendants of siblings of the affected child and the lack of a known mutation have finally ruled out the genetic etiology of this disease. FAH occurs together with jaundice, coagulopathy, slightly high transaminase levels (twice or thrice the normal value), high blood ferritin $(95 \%$ of patients have a ferritin level of approximately $800 \mathrm{ng} / \mathrm{ml}$ ), and high iron saturations values. Ferritin is an acute phase protein which is also increased in other causes of NALF, mainly viral hepatitis. Diagnosis is confirmed once extrahepatic iron deposits are observed through magnetic resonance imaging and/or oral cavity salivary gland biopsy. ${ }^{2-4}$

FAH treatment is aimed at the alloimmune etiology and consists in the combination of double volume exchange transfusion and highdose intravenous immunoglobulin to remove aggressive antibodies and block their action. This treatment should be implemented immediately if FAH is suspected. Diagnostic confirmation is very important in these patients because FAH is associated with a high rate of recurrence in future pregnancies and may be prevented by administering high-dose intravenous immunoglobulin to the pregnant mother. ${ }^{4-6}$

\section{Viral infections}

A perinatal herpes, enterovirus, adenovirus, or parvovirus infection may cause NALF relatively often $(20-30 \%)$. Diagnosis is confirmed with the polymerase chain reaction (PCR) test in blood, nasal secretions, or feces. Perinatal viral infection leads to NALF once it causes acute hepatic necrosis. In these cases, the transaminase level is very high; postmortem histopathological liver findings showed generalized necrosis, sometimes with parenchymal collapse. ${ }^{1,3}$
The most common virus is herpes simplex virus (HSV), which causes a broad range of clinical manifestations, from mucocutaneous involvement (skin, eyes, and mucous membranes) to neurological involvement (central nervous system), and even to disseminated disease. NALF may occur in the context of a disseminated or an isolated liver infection. In either case, the typical skin findings are not usually observed; for this reason, while viral results are pending, all patients should be treated with intravenous acyclovir.

The rate of neonatal HSV infection transmission during pregnancy is higher in the case of a primary infection (33-50\%), and transmission usually occurs at the time of delivery due to contact with genital secretions. Most neonatal herpes patients have no maternal history of genital herpes at the time of delivery or during pregnancy, or a sexual partner with a history of herpes. Newborn infants usually have fever, lethargy, food rejection, and bloating, with or without blisters, towards the end of the first week. The presence of seizures is a warning sign for neurological involvement. Prognosis depends on localization, and mortality ranges from $0 \%$ for the mucocutaneous presentation to $80 \%$ for the disseminated form in spite of treatment. In addition, the localized forms may progress to more severe forms if not treated, so therapy should not be delayed while diagnostic test results are pending. Recently, in response to systemic acyclovir routine use, the proportion of newborn infants with severe disseminated disease has decreased. The prognosis of NALF caused by HSV is unfavorable, and for this reason acyclovir should be indicated early. Post-transplant course may be favorable, even in children with active viral load, and requires long-term treatment with acyclovir. ${ }^{78}$

Enteroviruses are ribonucleic acid (RNA) viruses of the Picornaviridae family and, even though they cause a mild disease in adults, they may be the cause of NALF. A maternal history of recent respiratory disease or diarrhea must give rise to the suspicion of this etiology. Although most perinatal enterovirus infections are mild, some may result in NALF. The course of this disease in newborn infants is usually unfavorable. Treatment effectiveness is under discussion. High-dose immunoglobulin is recommended for newborn infants with severe infections; pleconaril, an investigational drug, may be used on a compassionate basis. ${ }^{1,2}$ 
A cytomegalovirus infection may be acquired in the prenatal or perinatal period, and is the most common congenital viral infection. Perinatal transmission is the result of exposure to infected cervical mucus secretions, breast milk, or blood products. It occurs in $0.2-2.2 \%$ of births worldwide and is caused by a maternal primary or recurrent infection. The clinically apparent neonatal disease is more likely to develop after primary maternal exposure, especially if it occurs during the first half of pregnancy. Maternal anti-cytomegalovirus antibodies are considered protective because they prevent infection or symptoms in term infants, but not in preterm infants, who lack them. In spite of the presence of hepatitis, progress to NALF is uncommon in this disease; only isolated reports have been made on this complication. ${ }^{1,2}$

\section{METABOLIC DISORDERS}

Metabolic disorders are a group of hereditary diseases caused by a defect or deficiency in one or more enzymes necessary for substrate metabolism, which triggers a toxic intermediate within the hepatocytes or the absence of a metabolite that is essential for their functioning. Metabolic disorders are rarely the cause of NALF. ${ }^{1-3}$

Galactosemia, type 1 tyrosinemia, and hereditary fructose intolerance -all inherited autosomal recessive disorders- may occur in the neonatal period together with jaundice or hypoglycemia, and may occasionally progress to NALF. Out of these three conditions, galactosemia may be more common in the first month of life, once milk feeding is introduced. Galactosemia is a disease caused by mutations in the GALT gene (9p13), which codes an enzyme called galactose1-phosphate uridylyltransferase, and these cause galactose, galactose-1-phosphate, and galactitol accumulation in the tissue of affected patients. The primary source of galactose is lactose, a component of breast milk and most infant formulas. When a newborn infant receives this food, they usually develop food rejection, growth failure, clinical signs of liver damage (jaundice, liver enlargement, coagulopathy, hypoglycemia), and cataracts, which are usually present at birth. In the absence of an adequate treatment (galactose restriction), in addition to NALF, patients may develop Gram-negative sepsis, and even die. In spite of treatment, long-term complications may occur, including cognitive impairment, motor deficits, ovarian impairment, e.g. impaired fertility, and reduced bone density caused by endogenous galactose production from glucose. In many countries, infants undergo a newborn screening for the purpose of identifying asymptomatic patients before severe damage occurs as a result of this devastating disease. However, knowing that transfused patients may have false negative results given that enzymatic activity is determined in red blood cells, it is necessary to rule out this condition. For this reason, it is essential to exclude galactose from the diet of any newborn infant with severe and progressive liver disease until diagnosis is confirmed. . $^{1,29-13}$

The clinical manifestations of hereditary fructose intolerance appear following the introduction of fructose or sucrose, which takes place once solid food is introduced in the newborn infant's diet. However, the administration of juices or syrups may lead to an early onset. . $^{9-12}$

In the case of a patient with NALF, it is recommended to confirm neonatal screening results to rule out galactosemia, register the infant's feeding history to exclude hereditary fructose intolerance, and determine the presence of succinylacetone in urine to rule out type 1 tyrosinemia. Although these conditions may exceptionally be the cause of NALF, test costs are minimal, considering that they are treatable diseases. ${ }^{1,2,9-12}$

Respiratory chain (RC) defects account for a group of mitochondrial diseases. These organelles are in charge of generating energy in the form of adenosine triphosphate (ATP), an element that is essential for effective cell functioning. The mitochondrial respiratory chain includes five complexes made up of, at least, 89 different subunits; of these, 13 are coded by the mitochondrial genome. Both deoxyribonucleic acid (DNA) depletion and mitochondrial RNA gene mutations may cause defects in the RC complexes.

The consequences of mitochondrial disease vary because defective mitochondrial DNA may have a heterogeneous body distribution, and each mutation is modulated by other genome variations. The mutation may cause hepatic disease in a child or brain involvement in another. A mitochondrial cytopathy may affect any organ or tissue; the most commonly affected ones are those that require more energy, such as the brain, a muscle, and the liver. NALF is a common finding in RC defects. In general, it occurs together with cholestasis, cirrhosis, and / or cytolysis quickly after birth or in the first weeks of life. 
Biochemical findings include a very high plasma lactate level, a high plasma lactate:pyruvate ratio ( $>20$, and frequently $>30)$, and increased beta-hydroxybutyrate: acetoacetate ratio in arterial blood (>2.0). The analysis of liver or muscle RC complexes generally shows decreased activity. Treatment is basically palliative, and includes vitamins, cofactors, respiratory substrates, or antioxidant compounds. None of these therapies has proven to be universally effective. Supportive treatment may also include sodium bicarbonate infusion during acute metabolic acidosis events, transfusions in the case of anemia and thrombocytopenia, and exogenous enzymes for patients with pancreatic insufficiency. ${ }^{1,2,12-14}$

A mutation in the TRMU gene (a protein involved in mitochondrial transfer RNA [tRNA] modifications) has been associated with NALF, which is sometimes transient. Patients who survive the initial episode may recover and grow normally. ${ }^{15}$

Defects in the urea cycle, mutations in the ornithine transcarbamylase gene, may be, in more severe cases, accompanied by signs of liver failure, which are corrected once accumulated ammonium levels are reduced. Acute events should be managed very quickly. Treatment consists in decreasing blood ammonium levels by discontinuing protein intake and administering intravenous glucose to reduce catabolism. In addition, the administration of oral or intravenous phenyl acetate or sodium benzoate is a biochemical alternative to achieve nitrogen excretion. Finally, direct ammonium removal may be required using high-flow hemodialysis. ${ }^{16}$

Liver transplantation (LT) should be indicated for RC defects only if the disease is exclusively restricted to the liver. It is necessary to consider that, even in patients with apparently isolated liver involvement, any other organ or system may be affected months or years later. ${ }^{1,2,17,18}$

\section{OTHER ETIOLOGIES}

Perinatal asphyxia or hypoxia usually alter liver function and progress to severe NALF in cases of asphyxia. This is part of multiorgan involvement caused by the deviation of blood flow from the skin and the splanchnic area to the heart, the adrenal glands, and the brain, intended to protect such vital organs against the lesion resulting from ischemic hypoxia. ${ }^{1,3}$

$\mathrm{HLH}$ is a clinical hyperinflammation syndrome that causes an ineffective immune response. It occurs as a result of inadequate $T$ cell and macrophage activation, which causes a defective apoptosis and reduced cytotoxic activity. There are a primary, familial, inherited autosomal recessive form and an acquired secondary form associated with systemic infection, malignancies, metabolic

TABLE 1. Characteristics of neonatal acute liver failure at the time of onset

\begin{tabular}{lccc}
\hline & Fetal alloimmune hepatitis & Viral hepatitis & Metabolic disorders \\
\hline History & & & No \\
Prematurity & ++++ & No & No \\
Oligohydramnios & ++++ & No & ++ \\
Intrauterine growth & ++++ & & No \\
Clinical manifestations & & No & ++ to +++ \\
Patent ductus arteriosus & ++++ & ++++ & + to +++ \\
Liver & + to ++ & ++ & Depending on the disease \\
Spleen & $+/-$ & Meningoencephalitis & $120-320$ \\
Other & - & & $40-140$ \\
& & $60-170$ & $4-5 \times \mathrm{N}$ \\
Lab tests & $8-17.6$ & $20-80$ & $1-2 \times \mathrm{N}$ \\
TB (mg/dL) & $2.3-6.4$ & $20-30 \times \mathrm{N}$ & $\cong 2.5$ \\
CB (mg/dL) & $1-2 \times \mathrm{N}$ & $2-3 \times \mathrm{N}$ & Yes (suspect \\
ALT/AST (IU/L) & $\mathrm{N}$ & $\cong 4.0$ & mitochondrial disease \\
GGT (IU/L) & $\cong 3.0$ & $\mathrm{No}$ & \\
INR & $\mathrm{No}$ & & \\
High lactic acid & & & \\
& & & \\
\hline
\end{tabular}

TB: total bilirubin; CB: conjugated bilirubin; ALT: alanine aminotransferase; AST: aspartate aminotransferase; GGT: gamma-glutamyl transferase; INR: international normalized ratio. 
disorders, or immune deficiency. The familial form is associated with gene mutations that code perforin proteins, Munc 13-4 and Syntaxin 11 (30-40\% of cases). Episodes are acute and similar to sepsis with fever, humoral inflammation, coagulopathy, and cytopenia. HLH should be suspected in an infant whose condition worsens in spite of the highest level of antimicrobial and supportive therapy. ${ }^{1-3}$

Neonatal sepsis, predominately caused by Gram-negative microorganisms, accounts for 25\% of mortality in developing countries. Liver failure is part of multiorgan dysfunction. ${ }^{1-3}$

Tables 1 and 2 show the characteristics of the main causes of NALF and a diagnostic proposal for this condition.

\section{MANAGEMENT OF NEONATAL ACUTE LIVER FAILURE}

The specific treatment for certain conditions has been described in the corresponding sections; however, in the case of an untreated disease or ineffective treatment due to a late diagnosis, LT should be proposed.
LT is particularly difficult at this stage of life because of the lack of clinical and biochemical parameters that would enable making a disease prognosis. In addition, the size of the liver to be transplanted, even if reduced, should be very small for it to fit into the newborn infant's abdominal cavity, therefore limiting the number of potential donors.

At this stage of life, the universally accepted principle should be applied: "a patient with ALF" should be transferred to a hospital where LT is an available therapeutic option. ${ }^{1-3,17,18}$

\section{REFERENCES}

1. Shanmugam nP, Bansal S, Greenough A, Verma A et al. Neonatal liver failure: aetiologies and management-state of the art. Eur J Pediatr 2011;170(5):573-81.

2. Taylor SA, Whitington PF. Neonatal acute liver failure. Clin Liver Dis 2016;22(5):677-85.

3. Sundaram SS, Alonso EM, Narkewicz MR, Zhang S, et al. Characterization and Outcomes of Young Infants with Acute Liver Failure. J Pediatr 2011; 159(5):813-8.

4. Costaguta A, Álvarez F. El nuevo paradigma de la hemocromatosis neonatal: hepatitis fetal aloinmunitaria. Arch Argent Pediatr 2012;110(3):237-43.

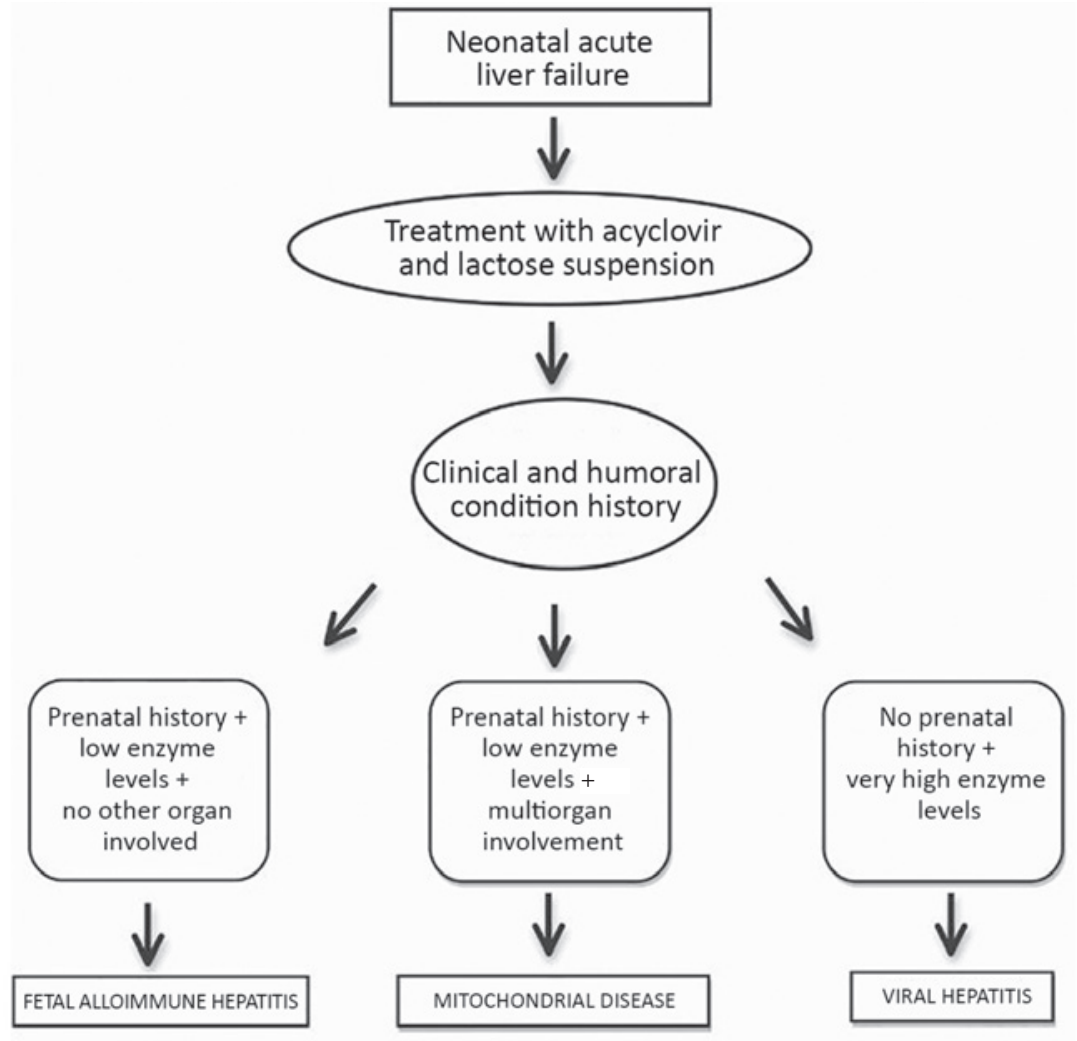


5. Rand EB, Karpen SJ, Kelly S, Mack CL, et al. Treatment of neonatal hemochromatosis with exchange transfusion and intravenous immunoglobulin. J Pediatr 2009;155(4):566-71.

6. Molera Busoms C, Quintero Bernabeu J, Martín del Carpi J. Hemocromatosis neonatal: otra entidad que deja de ser huérfana. Avances en el diagnóstico y manejo de la principal causa de fallo hepático agudo neonatal. An Pediatr (Barc) 2015;83(3):218.e1-3.

7. Verma A, Dhawan A, Zuckerman M, Hadzic n, et al. Neonatal herpes simplex virus infection presenting as acute liver failure: prevalent role of herpes simplex virus type I. J Pediatr Gastroenterol Nutr 2006;42(3):282-6.

8. McGoogan KE, Haafiz AB, González Peralta RP. Herpes Simplex Virus Hepatitis in Infants: Clinical Outcomes and Correlates of Disease Severity. J Pediatr 2011;159(4):608-11.

9. Saenz MS, Van Hove J, Scharer G. Neonatal liver failure: a genetic and metabolic perspective. Curr Opin Pediatr 2010;22(2):241-5

10. Mackay RJ, Bratkovic D, Couper R, Davidson GP, et al. Detection of treatable neonatal liver disease by expanded newborn screening. J Inherit Metab Dis 2008;31(Suppl 2):S271-3.

11. CroffieJM,GruptaSK,ChongSK,FitzgeraldJF.Tyrosinemia type 1 should be suspected in infants with severe coagulopathy even in the absence of other signs of liver failure. Pediatrics 1999;103(3):675-8.

12. Molleston JP, Sokol RJ, Karnsakul W, Miethke A, et al. Evaluation of the child with suspected mitochondrial liver disease. J Pediatr Gastroenterol Nutr 2013;57(3):269-76.

13. Champion MP. An approach to the diagnosis of inherited metabolic disease. Arch Dis Child Educ Pract Ed 2010;95(2): 40-6.

14. Lee WS, Sokol RJ. Mitochondrial Hepatopathies: Advances in Genetics and Pathogenesis. Hepatology 2007;45(6):1555-65.

15. Zeharia A, Shaag A, Pappo O, Mager-Heckel AM, et al. Acute Infantile Liver Failure Due to Mutations in the TRMU Gene. Am J Hum Genet 2009;85(3):401-7.

16. Gallagher RC, Lam C, Wong D, Cederbaum $S$, et al. Significant hepatic involvement in patients with ornithine transcarbamylase deficiency. J Pediatr 2014;164(4):720-25. e6.

17. Sundaram SS, Alonso EM, Whitington PF. Liver transplantation in neonates. Clin Liver Dis 2003;9(8):783-8.

18. Sheflin-Findling S, Annunziato RA, Chu J, Arvelakis A, et al. Liver transplantation for neonatal hemochromatosis: analysis of the UnOS database. Pediatr Transplant 2015;19(2):164-9. 